\title{
LEONARDO DA VINCI: DE LA ANATOMÍA A LA PINTURA
}

\section{María Bolaños}

\section{Domenico LAUREnZA, De figura umana. Fisiognomica, anatomia e arte in Leonar-} do, Florencia, Leo S. Olschki, 2001, 242 pp.

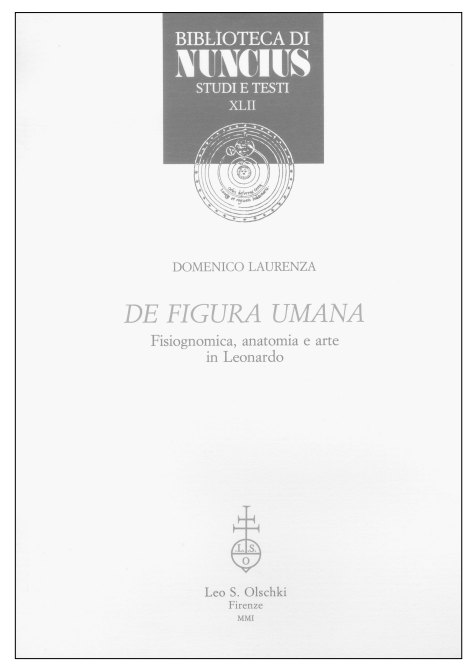

I. «Habría que ser como Leonardo para hablar de Leonardo». Esta advertencia con la que E. Gombrich iniciaba uno de sus artículos prueba la vigencia de un mito fabricado tempranamente, casi a la muerte del propio artista, cuando ya su biógrafo, Vasari (Vite), le definió como una figura «prodigiosa y celeste», como un afortunado sobre el que los cielos habían volcado todos los dones dándole ventajas inalcanzables: Leonardo fue admirable por su belleza corporal y su destreza física, por su imaginación incansable y su talento para el dibujo, por su rapidez intelectual y su paciencia investigadora, por el tierno amor por las criaturas vivientes y su desdén hacia los pedantes, por su sentido de la elegancia y su gusto burlesco, por su bondad y sus poderes mágicos. «Belleza, gracia y talento». Desde este primer, Leonardo siempre ha sido visto como un gigante que inspiraba asombro y temor, como una figura faústica e incomprensible. Un enigma. No sólo un sabio capaz de prever todas las invenciones del porvenir, un creador de extravagancias, ilusiones y máquinas extraordinarias, sino la esencia misma del poder del espíritu. Un hombre-enciclopedia: biólogo, geómetra, músico, óptico, mecánico, cosmólogo, ingeniero; un talento universal que lo auscultó todo, lo pensó todo, todo lo dibujó y lo numeró. Y lo hizo sin retórica, sin maneras, con una ingenua avidez de infinito, con una audacia y optimismo asombrosos, por lo que, más que la retórica del genio universal, le cuadran aquellos versos de Las flores del Mal en los que Baudelaire le presenta como un «espejo profundo y oscuro».

Sin embargo, esta leyenda actúa como un velo sobre el verdadero Leonardo, que aún hoy requiere ser rescatado de los tópicos irritantes en que le ha encerrado cierta historiografia vulgar, que le presenta como un creador cuya capacidad de invención carece de precedentes, de contextos o de deudas intelectuales. Una leyenda, por cierto, que él mismo había contribuido a forjar en ese célebre pasaje del Codex Atlanticus en el que se definía a sí mismo como un uomo senza lettere, frase en la que muchos han querido ver una antipatía por la cultura libresca y la orgullosa decisión de obedecer sólo a la inspiración de la naturaleza, aunque donde, quizá, no late en el fondo más que un lamento por su falta de familiaridad con el latín, una carencia, por lo demás, frecuente entre algunos artistas del Quattrocento.

Así que, por muy seductora que sea esa imagen del sabio rebelde que no le debe nada a nadie, del investigador inculto e intuitivo, los historiadores de la ciencia y de la cultura tienen la obligación de reconstruir el verdadero carácter del genio leonardiano en el suelo cultural en el que se gestó. Pues, aunque es cierta su rebelión contra la cultura tradicional, su denuncia de la inutilidad de gran parte de la escolástica y sobre todo su ardorosa defensa de la experiencia práctica, no lo es 
menos que, como ya señaló P. Duhem (Études sur Leonardo de Vinci. Ce qu'il a lu et ceux qui l'ont lu, 1913), las intuiciones más nuevas y más audaces de Leonardo estaban en buena parte inspiradas por la cultura científica medieval. Y que, tras el célebre empirismo leonardiano, y su indudable intuición, se esconden horas de lecturas científicas, y en particular su deuda con Nicolas de Cusa y con los físicos de la escuela tardomedieval de París.

Hay que tener presente, además, el poderoso influjo que sobre él hubieron de ejercer sus contemporáneos. La Florencia en que se formó el joven artista constituía a finales del siglo XV la más culta y avanzada capital europea, el centro más cosmopolita y sabio. Una nueva Atenas intelectual dominada en buena parte por la personalidad de Marsilio Ficino, cuyo influjo sobre la obra científica vinciana fue destacado por A. Chastel - primero en su Léonard et la culture y luego en su gran obra Art et Humanisme à Florence au temps de Laurent le Magnifique, 1959-: la idea del cosmos como un juego de fuerzas de diseño armónico, la presentación de las relaciones entre el alma y el cuerpo bajo una forma dramática, su defensa de la superioridad de la visión sobre cualquier otro modo de conocimiento, y, sobre todo, su visión analógica entre el microcosmos y macrocosmos, tantas veces expresada en sus escritos, como cuando dice que el cuerpo del hombre «encierra un lago de sangre donde, en la respiración, se dilatan y se contraen los pulmones», del mismo modo que «el cuerpo de la tierra tiene su océano que crece y decrece cada seis horas con la respiración del mundo».

Pero Florencia no era sólo un limbo neoplatónico de filósofos soñadores, y en la cultura de Leonardo, como ha insistido otro gran experto, E. Garin (Il problema delle fonte del pensiero di Leonardo, 1953), tiene un peso notable la otra Florencia: ese escenario de investigaciones experimentales, de discusiones aristotélicas (de un «aristotelismo actualizado y sutil»), cuyo influjo sobre las ideas anatómicas de Leonardo son palmarias, de debates lógico-físicos, de estudios médicos como los de Benivieni, de experimentos científicos como los de Toscanelli, de teorías arquitectónicas como las de Alberti, y de geógrafos y de reputados artesanos, cuyas habilidades, entre vulgares y herméticas, son tan importantes para comprender al Leonardo más empirista. Y era, sobre todo, la Florencia de filólogos como Valla y Poliziano, imbuidos de un gusto exquisito por la palabra, un gusto que reconocemos de inmediato en la «ansiosa ternura» con que Leonardo maneja la profunda humanidad del lenguaje, ya se trate de sus descripciones mecánicas, de las glosas añadidas a los márgenes de sus dibujos o de su Tratado de la pintura, obsesionado por dar con el término que mejor encarna su pensamiento, en todo su sabor y corporeidad.

Pero, probablemente, la singularidad de Leonardo, en este contexto renacentista de entre siglos, consiste no tanto en la amplitud de su horizonte mental, en la vastedad ilimitada de los campos que estudió y de las investigaciones que emprendió, sino en la visión unitaria y la honda coherencia de su universo mental. Una unidad que todos los especialistas, desde la tradición - de Vasari a Goethe- hasta los estudios recientes más relevantes, coinciden en resaltar, ya que Leonardo juzgaba a la pintura como la forma superior del conocimiento.

Desde esta perspectiva precisa hay que entender al Leonardo sabio e inventor y al uso personal que hizo de sus lecturas, búsquedas y observaciones científicas. Su ciencia no destaca por su rigor deductivo, ni por su sentido lógico. No establece métodos, ni fija cánones, ni organiza sistemas. Como recuerda Valéry en su honda Introduction à la méthode de Léonard de Vinci (1894), de los ingenios del Leonardo se desprende un encanto particular: el de parecer estar hechos pensando siempre en otra cosa. Ello se debe no sólo a su temperamento sino a que este corpus teórico multiforme hecho de lecturas apresuradas, de comentarios a vuelapluma, de anotaciones y reflexiones dificiles de ordenar, según ha probado Chastel, responde a la cultura de un artista, tal como ésta se entendía en los talleres florentinos del Quattrocento desde Brunelleschi, en un momento en que los artistas se afanaban por salir del marco medieval del ars mechanica y aspiraban a dominar los conocimientos propios de las artes liberales, si bien en Leonar- 


\section{ENSAYOS}

do este afán se caracterizó por una impaciencia y ambición obstinadas y sin precedentes. De ahí que su mayor aportación radique en la belleza, un poco desordenada, de sus intuiciones, en la agudeza de su penetración de la vida y la naturaleza, en su riqueza expresiva verbal y plástica, que dejan perplejo y, en el fondo, insatisfecho, al racionalista científico, pero colman la sensibilidad del hombre de gusto.

II. Este círculo leonardiano entre ciencia, naturaleza y pintura preside uno de las últimos estudios publicados sobre el artista, De Figura humana. Fisiognomica, anatomia e arte in Leonardo, editado en Florencia por el Instituto y Museo de Historia de la Ciencia (Leo S. Olschki, 2001), debido a Domenico Laurenza (Salerno, 1963). El autor, médico neuropsicólogo y miembro del Departamento de Estudios Histórico-Sociales y Filosóficos de la Universidad de Siena en su sede de Arezzo, ha desarrollado su trabajo como investigador en torno a la obra de Leonardo, fruto en buena parte de su estancia en el célebre Warburg Institute londinense. Su libro constituye una síntesis de su tesis doctoral y de algunos artículos y ponencias en congresos acerca de las relaciones entre anatomía, fisiognomía y pintura en la obra vinciana desde una perspectiva científica y médica, a partir del estudio de esa inmensa y a veces caótica masa de apuntes y dibujos de anatomía de Leonardo, dispersos por distintas colecciones (el Windsor Castle, la Albertina de Viena, la Biblioteca Ambrosiana de Milán o el Gabinete Nacional de Grabados y Dibujos de Roma).

Según él mismo advierte, el propósito de Laurenza, a diferencia de otros estudios que privilegian una clave de lectura de la fisonomía vinciana en términos psicológicos, como Caroli (Leonardo. Studi di Fisiognomica, 1991), o lingüísticos, caso de las interpretaciones semióticas de Courtine y Haroche (Histoire du visage. Exprimer et taire ses émotions, 1988) es profundizar en una línea de investigación esbozada anteriormente por algunos estudios clásicos - como los de Champfleury (Anatomie du laid d'après Léonard de Vinci, 1879), Hildebrandt (Leonardo da Vinci, 1927) o Baltrusaitis (Abérrations, 1957) - y mostrar la originalidad de Leonardo en la medida en que sus «esbozos de fisiognómica - esto es, el estudio de la forma externa del cuerpo como expresión del carácter-, forman parte de una investigación más general sobre la forma somática, tanto en su dimensión anatómica como en la artística».

Pues aunque la conexión entre anatomía y fisiognómica no sea una invención leonardiana, sino un aspecto del pensamiento biológico escolástico — y así lo prueba, por ejemplo, la inclusión de la Embriología en su visión de la Anatomía, siguiendo en esto a Alberto Magno en De animalibus- y aunque, de hecho, en su época, la Fisiognómica fuese ya una ciencia integrada en la enseñanza universitaria desde el siglo XIII, fue Leonardo el primero en inventar las imágenes y las representaciones visuales de esta ciencia, ya que ninguno de los tratados precedentes (a diferencia de lo que sucede con sus estudios tecnológicos o mecánicos, de los que sí existen abundantes precedentes iconográficos medievales) va más allá de la información escrita y verbal. Es ésta creación de un «discurso dibujado» la que otorga una dimensión fundadora y paradigmática a la obra leonardesca.

El estudio de Laurenza se inscribe en el grupo de todos aquellos que han defendido la importancia que la forma tiene en el método de Leonardo, para quien la relación entre el hombre y el mundo y el verdadero significado de la actividad humana pasan necesariamente por la sensibilidad. Frente a la esterilidad de la pura especulación mental, a Leonardo - y así supo verlo K. Clark en su ya clásico estudio sobre el pintor, Leonardo da Vinci. An Account of his Development as an Artist, 1939-, lo que le interesa es la relación entre el ver y el saber, una relación circular que parte del ojo, atraviesa la piel de la naturaleza, llega a sus fenómenos y procesos más recónditos y desde ahí vuelve al cerebro, a la razón especulativa, al número, a la mente que actúa como una fuerza mediadora. El primer instrumento de Leonardo es, pues, su ojo, que le permite plasmar en formas una imagen que resume la profundidad del universo, los misteriosos comportamientos de los seres y las cosas. En su modelo científico el dibujo es mucho más que una compañía 
refrescante de la idea, más que una ilustración del pensamiento abstracto. Leonardo no puede analizar cómo se cierra un muelle, cómo se dobla un paño de lana, cómo se comprime el aire bajo las alas de un pájaro, como se horada una montaña o se traslada un edificio entero sin derruirlo, sin trazar la forma visual que le permite anatomizar esa realidad para descubrir su mecanismo, penetrar en la estructura maquinal del fenómeno o del objeto y presentarlo como un juego de fuerzas secretas pero luminosamente claras. Es en la pintura donde el despiece de la naturaleza cristaliza en una imagen unitaria, se organiza y se carga de sentido. Ahí reside lo que Leonardo llamó la deità dell'arte, porque la capacidad de la pintura de penetrar en lo real y comprenderlo sólo es equiparable a la inteligencia divina.

Es en este contexto donde encuentra sentido su empirismo: cuando el artista defiende la experiencia frente a la autoridad, las manos frente a la mente, la actividad práctica frente a la especulación, la naturaleza frente a la Antigüedad, no es tanto por definirse en contra de la erudición humanista y de la cultura literaria como para ensalzar la superioridad de la pintura, que es, para él una ciencia suprema que representa la superación del momento puramente mental, un método según el cual el proceso de pensar se desarrolla en forma de imaginar, para obtener una visión unitaria de las cosas en su estructura profunda: la pintura, dirá, «separa los elementos esenciales de la naturaleza».

Y es que las investigaciones de Leonardo, su universo mental no puede por menos que remitirnos a esa «mirada terrestre» que persigue el arte renacentista, empeñado en reconstruir, con la mayor de las firmezas, la realidad sensible. El principio según el cual el arte debe ser una imitación de la vida humana, y abandona su propósito medieval de ser el símbolo de lo trascendente para reducirse a lo visible, se convertirá en un programa al cual irán destinados todos los esfuerzos de su generación, desde el tratamiento geométrico del espacio, esa ardua «conquista del horizonte» - a la que Leonardo contribuiría con una decisiva aportación, la perspectiva aérea, enseñando a los pintores a representar la sustancia atmosférica, el intervalo entre las cosas, la consistencia del vacío-, hasta la doctrina de la conveniencia — tal como ha demostrado R.W. Lee, en Ut Pictura Poesis, 1967—, según la cual cada objeto, figura y situación, para parecer reales, debían atenerse a rasgos característicos de edad, rango, circunstancia geográfica o contexto histórico, evitando desviaciones, anomalías, o improbabilidades: un pequeño torso no puede sostener una gran cabeza, Ifigenia no podía tener manos de vieja, César no puede llevar turbante y un rey debe ser barbudo y grave en su aspecto y su indumentaria. Es decir, una búsqueda científica de lo tópico, del esquema fijo, de la norma, a la que la contribución de Leonardo será de una relevancia decisiva. El ardiente interés con que se plantea la cuestión fisiognómica se inscribe en este marco de la representación natural, como por ejemplo, cuando estudia el llanto, sus variedades y los movimientos corporales que lo acompañan: el lloro desesperado, el sollozo sofocado, el gesticulante y gritador, el que sólo derrama lágrimas, el que esconde el rostro sobre el pecho, el que alza la cabeza al cielo, el que contrae las cejas y arruga las comisuras de la boca.

Esta extraordinaria relación entre el ver y el saber, entre pensamiento y percepción, entre idea y dibujo alcanza una de sus cumbres en los estudios fisionómicos analizados por Laurenza, que trata así de probar su novedad, buscando las conexiones entre arte y ciencia, y su convergencia en la indagación sobre los procesos de generación de la forma, entendida ésta en su sentido aristotélico, esto es, como la expresión de una finalidad o causalidad interna. Ya Vasari se había admirado de las anatomías humanas de Leonardo, de su gran habilidad para pintar osamentas, tendones y venas, de la bravura muscular de los caballos en su cartón para la Batalla de Anghiari y de cómo «palpitan los pulsos en el hoyo de la garganta» de Monna Lisa.

Según Laurenza se distancia aquí Leonardo no sólo de la tradición precedente, sino del futuro $D e$ humani corporis fabrica de Vesalio, que, basado en un método analítico y diseccionador, excluye la fisionomía de su modelo corporal. Frente a éste, prevalece en Leonardo un modelo sintético y compositivo, en el que lo que cuenta es la reciprocidad de las partes y de los sistemas somáticos, es decir, la 


\section{ENSAYOS}

figura, entendida no como una mera descripción anatómica objetiva de la morfología corpórea, sino como un cuerpo animado, habitado por un alma orgánica que lo hace actuar y vivir. Y esta visión tiene su continuidad e incluso su meta natural en el arte, en el que la figura pintada o esculpida es el punto de llegada, la síntesis final.

III. De Figura humana se divide en dos partes bien diferenciadas. En la primera, ordenada según un enfoque diacrónico, Laurenza recorre, en diversos subcapítulos, los tres momentos en los que, en el curso de su vida, Leonardo se interesó con una intensidad y dedicación especiales por temas somáticos, en sus vertientes anatómica, fisionómica y artística. El más precoz, al que dedica el primer capítulo, es su aproximación juvenil de 1478, dedicada al estudio artístico de la expresión facial, en unos pocos dibujos pertenecientes a la colección del Windsor Castle. Es aún el Leonardo florentino, de veintitantos años, aún muy influido por las enseñanzas adquiridas junto a su maestro Verrocchio. De esta misma época, datan algunos célebres dibujos, los del viril adulto que encarna el vigor y la firmeza, y los del efebo afeminado, que inspiraron las interpretaciones psicológicas de Gombrich y Clark. Laurenza se interesa, en cambio, por algunos otros dibujos marginales del mismo período, en los que, a su juicio, se atisba una primera tentativa de reflexión fisionómica sobre la expresión del coraje o de la apatía, libres de toda inmediata intención artística, en la que, por ejemplo, Leonardo, siguiendo un procedimiento propio de la escolástica que permanecerá residualmente en la ciencia renacentista, individualiza un signo, la prominencia de la frente, como revelación de una analogía, el coraje del león, que remite a una expresión típica: la fisonomía del colérico.

La segunda etapa, más extensa y sistemática, especialmente intensa en los años en torno a 1489, tiene lugar cuando el artista está ya instalado en Milán al servicio de Ludovico el Moro. Laurenza se centra en el estudio de los dibujos craneales, sobre los que ya otro gran especialista, M. Kemp, había demostrado (Disection and Divinity in Leonardo's Late Anatomies, 1972), su pertenencia a una tradición concerniente a la investigación sobre la sede del alma, si bien Kemp había formulado un juicio muy negativo acerca de la, según él, escasa originalidad de Leonardo como anatomista. Laurenza reconduce las indagaciones vincianas a un contexto distinto, que no es el de la anatomía descriptiva - vesaliana, por tanto-, sino a una anatomía funcionalista y sintética que saca a su objeto de los estrechos límites de la escolástica médica para proyectarlo sobre un horizonte conceptual más amplio, en el que también cuenta la mirada del artista.

Pues en Leonardo, la búsqueda del alma, escasamente metafísica, está siempre asociada a un principio orgánico, íntimamente conectada con el cuerpo, sus órganos y sus funciones. De ahí su interés por localizar y «dibujar» el sentido común, al que identifica con el alma en su conjunto: Leonardo diseña sus relaciones con el movimiento muscular voluntario, su dependencia de la estructura maxilar, su precisa ubicación en el ventrículo intermedio, su representación como un punto. Pero lo importante de estos detallados estudios es su conexión con sus intereses fisionómicos, como lo prueba la figuración obsesiva en estos años de una imagen predilecta, el hombre leonino, su insistencia en la protuberancia de la frente que le caracteriza, y sobre el carácter iracundo de quien porta sobre el rostro esa «nube supraciliar» ya descrita por la Fisiognomica pseudoaristotélica - y que, por cierto, identificamos también en el David miguelangelesco-, en la que él ve una muy precisa estructura ósea, el seno frontal, que conecta con el sentido del olfato, y, de paso, con cierto tipo de brutos, de «uomini bestiali, e con poca ragione».

En razón de su semejanza genérica, estos estudios craneales son puestos en relación por Laurenza con algunas de las pinturas contemporáneas más brillantes, como el retrato de Cecilia Gallerani, la favorita de Ludovico el Moro, o la misteriosa Belle Ferronière, cuyas cabezas, de extraordinaria belleza, se sostienen en el espacio oscuro que las circunda con una tectónica sólida, regular y hermosamen- 
te nucleada (aunque la entalladura ósea, la frente alta y la rudeza de las manos de sus retratadas escondan un canon casi masculino de belleza - con esa belleza varonil, quieta y severa, propia del gusto clásico-). Confluencia que revela cómo unos y otros, dibujos anatómicos y retratos femeninos, comparten una misma reflexión sobre la forma, entendida ésta no como un recubrimiento de la materia, como la epidermis de un cuerpo, sino pensada siempre a partir de un interior causal y natural.

En el tercer capítulo de esta primera sección, Cardiocentrismo, el autor aborda la última etapa en el pensamiento anatómico vinciano, dominado, como han señalado Garin y Chastel, por una visión maquinal de la anatomía humana, y del universo en general, que se afirma de manera creciente en la obra de Leonardo desde 1500, fecha de su regreso a Florencia en un momento en que su carrera está en lo más alto y su prestigio es inmenso: realiza el cartón de Santa Ana, la Virgen y el Niño, creando en la ciudad sorpresa y provocación, trabaja en el proyecto de su libro sobre la pintura, pinta La Gioconda.

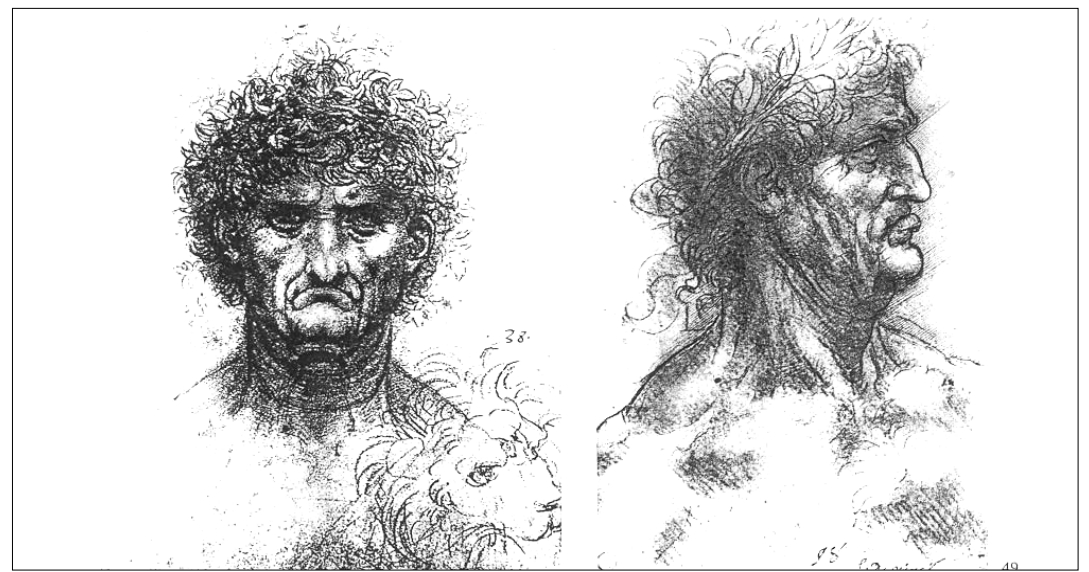

Laurenza analiza detalladamente los dibujos realizados en torno a 1504-1509, en los que Leonardo da primacía al corazón como centro vital, defendiendo con ello una visión más fluida y dinámica del cuerpo humano, reducible al modelo de la máquina y al juego de movimientos y de las fuerzas: el ojo del pintor descubre canales y flujos, palancas y pesos, motores y empujes, cifras y proporciones. Frente a los estudios craneales que expresaban una concepción dura, ósea del cuerpo, una forma duradera y estable, en la que primaba la arquitectura del esqueleto, en la madurez de Leonardo se aprecia una evolución hacia las morfologías corpóreas más blandas y variables que, como decía Aristóteles, no tienen un punto final de crecimiento, a diferencia del esqueleto, la fábrica del cuerpo, que, llegada una cierta edad, alcanza el límite de su grandeza. La sede anatómica de este nuevo horizonte vitalista es el corazón. Leonardo estudia el corazón como músculo, su funcionamiento neumático, como una estufa, su relación con la producción de esperma, con la fuerza y los músculos, con el origen de las venas, con el calor corpóreo y con los spiriti vitali, responsables del mantenimiento de la vida. Es en este órgano en el que muchos clásicos, desde Aristóteles, habían hecho residir las pasiones, que actúan sobre el cuerpo según un mecanismo cardiocéntrico, termodinámico y neumático, que se expresa por medio de lágrimas, temblor y fiebre. No es, pues, casual que la creciente atención que Leonardo reserva al corazón en sus estudios anatómicos coincida por esas mismas fechas con su interés por las pasiones, una exploración intensificada con motivo de los estudios destinados a su Battaglia d'Anghiari, para la Sala del Consejo de Florencia 


\section{ENSAYOS}

realizada entre 1503 y 1506 (del mismo modo que se entrelazan —en esa primera década del Quinientos - la búsqueda de un modelo venusino de belleza ideal, para los bocetos de la Leda, y los estudios anatómicos sobre el cuerpo de la mujer y sus órganos genitales). Pero Laurenza de acuerdo con su hipótesis proyecta este universo anatómico sobre el campo de la fisiognomía al asociar la fisiología cardiaca con el temperamento y la forma del cabello — así, el cabello crespo como síntoma de audacia y caracterización del tipo heroico-leonino-.

La parte II de De Figura humana abandona el enfoque cronológico y Laurenza se centra en algunos argumentos específicos sobre la forma del cuerpo, como las consecuencias fisiognómicas de la componente muscular y sus efectos iconográficos, en los retratos o en los cuadros de escenas. Con la excepción del célebre dibujo veneciano de 1490, en el que, siguiendo a Vitruvio, Leonardo dibuja el cuerpo humano como una abstracción intemporal matemática, como un hombre inalterable y sin edad, los restantes dibujos vincianos aplican un modelo patognómico, de raíz médica tardoescolástica, según el cual las pasiones más constantes en un individuo terminan por modificar su aspecto, una idea que deriva de una concepción del cuerpo humano como una estructura mutante que se altera en el curso de la vida, sea por vía natural (pérdida del cabello, arrugas), sea por accidente (la fatiga, el llanto, la ira, la enfermedad), Al concebir el cuerpo como una materia cualitativa, que es «pura naturaleza» y, por tanto, inmedible matemáticamente, se desentiende del canon y de toda morfología idealista y se interesa por estudiar, por ejemplo, cómo se puede descifrar el ánimo de una persona por los signos que dejan impresos en su cuerpo las pasiones, o bien, y éste es un tema recurrente, casi obsesivo en Leonardo, el devastamiento somático y espiritual producido por la vejez: sus dibujos de ancianos, como la sanguina de Windsor, describen, con una respetuosa dignidad, cómo los músculos se sutilizan, las arterias se ocluyen, la piel se separa de la carne y las líneas de la frente ocultan lamentos y pensamientos tristes.

Para sintetizar este modus operandi de Leonardo, Laurenza concluye dedicando los dos capítulos finales a dos grandes obras del pintor, la Cena del refectorio del convento milanés de Santa Maria delle Grazie, y la Battaglia d'Anghiari. En el primero analiza el modo en que Leonardo describe, siguiendo una práctica usual en los talleres artísticos de la Florencia de la segunda mitad del Quattrocento, el ethos de cada uno de los apóstoles, es decir su tipo psico-somático, siguiendo la tradición hagiográfica e iconográfica que les hace, uno a uno, diferentes y reconocibles. Pero profundiza paralelamente en el pathos de la escena, esto es, las emociones momentáneas de los actores - lo que Leonardo llamó «accidenti mentali» y Alberti «movimenti d'animo»-, en el preciso momento en que Jesús les advierte que sabe que uno de los presentes le ha traicionado, desencadenando en sus acompañantes un nudo de reacciones variadas: admiración, incredulidad, dolor, miedo, nerviosismo o ira. Y no deja de ser interesante, como señaló Gombrich, comprobar hasta qué punto el pintor describe el impacto casi físico que una palabra produce en un grupo de personas, que se mueven, retroceden o se adelantan con esa mismo materialidad dinámica con la que, Leonardo había dibujado la propagación del movimiento en las aguas o los circuitos de la sangre en el cuerpo, traduciendo su gusto y su curiosidad por lo entrelazado, por los efectos de resonancia o transmisión, que inspiran buena parte de la sensibilidad del artista («el agua percutida por el agua produce círculos alrededor del punto percutido»).

En la Cena, Leonardo, que aborda la escena sagrada partiendo de un arduo trabajo teórico, de consulta de fuentes, encaja la tradición iconográfica con el tipo humano que él se había imaginado y las reacciones más acordes con su temperamento, desplegando todo un tratado del comportamiento humano a través de su dominio de la representación anatómico-fisiognómico: reencontramos así las cabezas tectónicas de sus estudios craneológicos en las figuras de Pedro, Andrés y Simón, el enrojecimiento de la piel como efecto de una emoción súbita en Santiago el Mayor, la antítesis fisiognómica entre el carácter contemplativo de Juan y el iracundo de Pedro, el gesto de Mateo, hombre de cultura refinada, o el sufrimiento contenido del tímido Andrés. 
El libro concluye con un capítulo sobre los hombres bestiales, un tema esencial para conocer el pensamiento de Leonardo, para el cual la comparación entre el hombre y el animal adquiere una radicalidad que va más allá de las premisas aristotélicas y escolásticas, en tanto que traspasa la frontera decisiva que va de la analogía a la continuidad. Las analogías se ponen de manifiesto mediante diversos recursos: mediante alegorías (el armiño, emblema de la moderación de Cecilia Gallerani) mediante afinidades morfológicas (el hombre airado semejante al león en la estructura de la cabeza y el cabello). Pero, más allá de esas similitudes entre el hombre y la bestia, a Leonardo le atraen las naturalezas intermedias, las malformaciones anatómicas, la potencial contaminación entre humano y animal, que le llevan a plantear la diferencia entre hombre y león, o entre hombre y perro, como una cuestión de grado, cuantitativa, de variación entre «larguras y grosores», negando en cierto modo, la especificidad de la naturaleza humana, su parte divina. Y aunque, indudablemente, el planteamiento leonardesco tiene un valor instrumental, en tanto que le permite conocer mejor, por extrapolación, la naturaleza del hombre, según Laurenza, sus investigaciones parecen adherirse a menudo a una línea de pensamiento biológico, a un programa de anatomía comparada, donde el estudio de las especies animales adquiere valor autónomo.

La máxima expresión artística de la potencia bestial del hombre es la Battaglia d'Anghiari y sus estudios preparatorios, un tema recurrente que le atrae y que aparece aludido en su Tratatto della Pittura en el que habla de una «batalla sangrienta, en medio de sombras oscuras y tenebrosas, envueltas por la humareda de máquinas terribles y asesinas, por la polvareda que levantan los enloquecidos desgraciados temblando de horrible muerte». La guerra era, además, para él, una experiencia familiar. Desde 1502 estaba al servicio de Cesar Borgia como arquitecto, inspector de fortificaciones e ingeniero general y lo había acompañado en la campaña de la Romaña; cuando recibe el encargo de esta obra, acababa de contemplar con sus propios ojos el asedio de Pisa, con vistas a desviar el curso del Arno. La ferocidad de la batalla, «pazzia bestialissima», le permite combinar un doble registro temático: por un lado, sus ideas acerca de las nociones de vórtice y remolino, un tema científico que había abordado extensamente en el curso de su vida, por ejemplo, cuando estudia el impacto del agua sobre el agua, con sus choques y turbulencias, y, por otro, su obstinado interés por las pasiones humanas. La batalla es el tema ideal para escenificar la conexión entre el hombre y el animal, la caída del hombre en su parte más bestial, el modo en que los guerreros se funden como centauros con sus caballerías (una imagen que Isidoro de Sevilla había expuesto en sus Etimologías, fuente indudable de Leonardo), y éstas participan de la ferocidad rabiosa de los soldados. Esta empatía emocional es también anatómica como explica en un dibujo preparatorio de la batalla, en el que añade la siguiente nota: «para equiparar la osamenta del caballo y la del hombre, en el dibujo de las piernas, colocaré a éste de puntillas».

En el tratamiento de esta escena, de la que desgraciadamente no conservamos el original, se revela en todo su alcance el secreto de la inteligencia leonardiana, su capacidad de ver las conexiones entre cosas, de atrapar la ley de la continuidad que se le escapa al hombre común y llevarla al límite de su tendencia en un vértigo de analogías. Para Leonardo la batalla se resuelve en nudos, caídas, choques, torbellinos y velocidades de una infinita complejidad, gestada en ese ostinato rigore que fue siempre su divisa. Dice Valéry que algunos hombres sienten con especial delicadeza el placer de la individualidad de los objetos y se entretienen hipnotizados en el ángulo de una mesa o en la sombra de una hoja, rescatando lo que tienen de seres únicos. La imaginación de Leonardo, por el contrario, se ejerce en la fluidez de los estados transitorios, de los agrupamientos inconstantes, de los cambios, los movimientos y las precipitaciones. Como si las variaciones de las cosas le pareciesen demasiado lentas cuando están en calma, adora las batallas, las tempestades, la lluvia y los hombres volantes. En los últimos años, su mente se vio más y más invadida por sentimientos de decadencia, y catástrofe, y de esa etapa final datan sus más famosos dibujos del Diluvio Universal y del fin del mundo, que tanto interés despertaron entre los artistas del siglo XX. 